\title{
What is the optimal glaucoma treatment: reducing aqueous humour production or facilitating its outflow?
}

\author{
Hanspeter E. Killer ${ }^{1,2} \cdot$ Achmed Pircher $^{3}$ \\ Received: 31 January 2020 / Accepted: 24 March 2020 / Published online: 15 May 2020 \\ (c) The Royal College of Ophthalmologists 2020
}

Glaucoma is an optic neuropathy characterized by progressive damage to axons and loss of retinal ganglion cells. It is the most frequent disease of the optic nerve and the second leading cause for blindness in the world [1]. Elevated intraocular pressure (IOP) is a recognized risk factor for the largest group of glaucomatous optic neuropathies, including primary open angle glaucoma (POAG), pseudoexfoliation glaucoma, angle-and pupillary block glaucoma (with intermittent increase of IOP), as well as a group of secondary glaucomas that include neovascular glaucoma, traumatic and uveitic glaucoma. Common to all these forms are either constant or intermittent elevation of IOP.

The complete pathogenesis of glaucoma seems not yet to be comprehensively elucidated. In addition to high IOP glaucoma groups, there exists a subgroup of POAG called normal tension glaucoma (NTG) in which IOP is either normal or even within the lower normal range. In high-tension glaucoma, elevated IOP is an established risk factor. However, in NTG, which comprises up to $30 \%$ of glaucoma in the western hemisphere and up to $90 \%$ in the far east, its significance is still to be better defined $[2,3]$.

The fact that IOP can differ so substantially between glaucoma groups encourages the search for additional mechanisms that may also lead to glaucomatous optic disc pathology and visual field damage.

Hanspeter E. Killer

killer@ksa.ch

1 Department of Ophthalmology, Cantonal Hospital Aarau, Aarau, Switzerland

2 Department of Biomedicine, Ocular Pharmacology and Physiology, University Hospital Basel, Basel, Switzerland

3 Department of Neuroscience/Ophthalmology, Uppsala University, Uppsala, Sweden

\section{Other mechanisms than high IOP involved in the pathophysiology of glaucoma}

A recently discussed potential risk factor which may broaden the role of IOP in NTG is the so-called translaminar pressure gradient, which is defined as TLP $=$ IOP-CSFp [4]. Although interesting from a mechanical standpoint, this concept still lacks critical data, such as the local cerebrospinal fluid (CSF) pressure behind the lamina cribrosa. Additionally needed are studies that measure simultaneously IOP and CSFp behind the lamina cribrosa [5, 6]. Given the complex anatomy of the subarachnoid space of the optic nerve and the frequent observation of its kinking, the data available today are not informative enough to build a model and to draw valid conclusions.

Other risk factors for POAG include vascular dysregulation [7] and compartmentation of the subarachnoidal space of the optic nerve [8]. Recent research onto the pathogenesis of glaucoma focused on the composition and role of CSF. In a cohort of NTG patients, we demonstrated by using computer assisted tomography and MRI assisted flow studies that there were impaired CSF dynamics within the subarachnoid space of the optic nerve [9, 10]. CSF turnover impairment was most pronounced in the optic nerve portion behind the globe, the so-called bulbar region of the optic nerve [11].

\section{The role of CSF and the link between glaucoma and Alzheimer}

Recent reports indicate that glaucoma development has a major neurodegenerative component that is probably linked to parallel pathogenetic processes as occurring in Alzheimer's disease. CSF fulfils a variety of functions including the nutrition of neurons and axons and the removal of toxic substances and biological debris [12]. Impaired CSF dynamics in the subarachnoid space of the optic nerve could therefore lead to an accumulation of waste products which 
may have toxic effects on the axons of the optic nerve [13]. Reduced turnover of the CSF with accumulation of ABetaand Tauprotein levels is considered to be one of the risk factors in Alzheimer's disease [14, 15]. There is quite possibly a link between glaucoma and Alzheimer's [16, 17] and impaired CSF dynamics and stagnation of CSF with accumulation of toxic proteins might be a common pathogenetic feature. In the case of glaucoma an abnormal accumulation of potentially toxic proteins was found not only in the subarachnoid space of the optic nerve but also in the aqueous humour (AH) of the anterior chamber. The protein content measured in the $\mathrm{AH}$ of glaucoma versus non glaucoma patients has demonstrated significant differences $[18,19]$.

\section{Lowering IOP and its impact onto the velocity of aqueous humour}

Lowering IOP is the only generally accepted treatment of glaucoma today. Lowering IOP is thought to result in a reduction of a pressure mediated damage to retinal ganglion cells and axons. This concept however is exclusively mechanical. Assuming that pressure (mechanical force), is the exclusive pathophysiological mechanism leading to glaucoma, lowering pressure would be expected to stop the progression of glaucoma.

Focussing exclusively on the IOP reduction is inadequate in face of the complexity of this sight threatening disease in which $30-90 \%$ of the patients have no elevated IOP and in whom visual field defects occur and/or progress despite a low IOP [2, 3]. In addition, there is a substantial number of high-tension glaucoma patients in whom the disease progresses in spite of effectively lowered IOP.

Given the equation: Glaucoma = high IOP, glaucoma should not manifest if IOP is not elevated. What about looking at the other side of pressure, mainly velocity. According to Bernoullis equation, pressure is inevitably linked to velocity $\rho \frac{v^{2}}{2}+p=c$. Based on this relationship, lowering of pressure will result in improving velocity of the fluid in question. This relates to CSF in the subarachnoid space of the optic nerve as well as to $\mathrm{AH}$ in the anterior chamber of the eye [20].

\section{Aqueous humour and its impact in glaucoma: is it only pressure?}

The source of AH is the ciliary body. AH consists of water and an impressive proteome of at least 2851 proteins. There is a large overlap of proteins between plasma and ciliary body but 896 proteins were found to be unique in the ciliary body [21]. AH can flow from the posterior chamber into the anterior chamber and can also freely diffuse into the posterior cavity to the retina [22]. If the turnover of $\mathrm{AH}$ is compromised because of a partially blocked outflow path (trabecular meshwork, collector channels and Shlemn's canal) the pressure in the anterior chamber and the eye rises. Along with the increase of pressure the velocity of the fluid is being reduced (most likely in a non linear fashion) that results in a pressure mediated reduction of CSF turnover. Thus, an increase in the concentration of proteins, some of them toxic, is to be expected. Possible candidates of such noxious proteins are cystatin $\mathrm{C}$ that inhibits lysosome action and caspase 14 precursor that can induce apoptosis [23]. If the concentration of such proteins enhances, they can diffuse into the posterior cavity up to the retina where they can exercise their effect onto the retinal ganglion cells and axons.

Interestingly a study comparing the concentration of betatrace protein in $\mathrm{AH}$ of cataract and glaucoma patients demonstrated a larger concentration of betatrace in the $\mathrm{AH}$ in the glaucoma group [18]. The role of betatrace protein is manyfold as it is a prostaglandin synthetase. It acts as a chaperon as well as an inducer of apoptosis. Whether it has a beneficial effect or a toxic one seems to be depending on the receptor pathway it signals to target cells [24, 25]. In a different study in glaucoma patients differentially expressed proteins were found that are involved in cholesterol-related, inflammatory, metabolic, antioxidant and proteolysis-related processes [19].

\section{Glaucoma therapies and their mechanisms of action}

Although glaucoma treatment aims exclusively at lowering IOP there are currently mainly two treatment strategies available: topical or systemic medication and surgical shunt procedures. There are several different classes of pressurelowering medications available with different mechanisms of action. While prostaglandins and cholinergic agonists effectuate an increase of the $\mathrm{AH}$ outflow, carbonic anhydrase inhibitors, $\alpha$-adrenergic agonists and $\beta$-adrenergic blockers cause a reduction of the AH production. Their effect on the proteome of the $\mathrm{AH}$ is not yet understood.

Surgical procedures, except cyclodestructive procedures target to lower IOP by shunting $\mathrm{AH}$ from the anterior chamber under the conjunctiva or in the pre-existing outflow routes. The surgical spectrum for this purpose is wide, and many different techniques and devices are in use. While laser surgery (ALT, SLT) increases the outflow by focusing on the trabecular meshwork, incisional surgery (trabeculectomy, deep sclerectomy, viscocanalostomy, canaloplasty and aqueous shunt devices (MIGS) allow the AH to bypass the trabecular meshwork through an artificially created 
canal or an installed tube. They do not interfere with the proteome of the $\mathrm{AH}$ while the effect onto the proteome with drop medication is not determined.

\section{Conclusion}

There is evidence that aggregation of proteins and different biochemical processes in the CSF as well as in the AH is likely to be involved in the pathogenesis of glaucoma. Therefore, a treatment option that facilitates the outflow of $\mathrm{AH}$ from the anterior chamber might not only be beneficial for reducing IOP but also for the washout of potentially noxious proteins. If we consider glaucomatous damage something more as just a mechanical damage to ganglion cells and retinal axons we hypothesize that interventions focusing on the $\mathrm{AH}$ outflow might be preferable to interventions that focus on the reduction of $\mathrm{AH}$ production.

Acknowledgements Thanks to Dr J Stekhoven for helpful comments on the manuscript.

\section{Compliance with ethical standards}

Conflict of interest The authors declare that they have no conflict of interest.

Publisher's note Springer Nature remains neutral with regard to jurisdictional claims in published maps and institutional affiliations.

\section{References}

1. Thylefors B, Negrel AD. The global impact of glaucoma. Bull World Health Organ. 1994;72:323-6.

2. Cho HK, Kee C. Population-based glaucoma prevalence studies in Asians. Surv Ophthalmol. 2014;59:434-47.

3. Klein BE, Klein R, Sponsel WE, et al. Prevalence of glaucoma. the Beaver Dam Eye Study. Ophthalmology. 1992;99:1499-504.

4. Jonas JB, Wang N, Yang D. Translamina cribrosa pressure difference as potential element in the pathogenesis of glaucomatous optic neuropathy. Asia Pac J Ophthalmol. 2016;5:5-10.

5. Killer HE, Pircher B. TLP: a premature concept. Eye. 2016; 30:166-7.

6. Linden C, Qvarlander S, Johannesson G, et al. Normal-tension glaucoma has normal intracranial pressure: a prospective study of intracranial pressure and intraocular pressure in different body positions. Ophthalmology. 2018;125:361-8.
7. Yamazaki Y, Drance SM. The relationship between progression of visual field defects and retrobulbar circulation in patients with glaucoma. Am J Ophthalmol. 1997;124:287-95.

8. Killer HE, Subramanian PS. Compartmentalized cerebrospinal fluid. Int Ophthalmol Clin. 2014;54:95-102.

9. Killer HE, Miller NR, Flammer J, et al. Cerebrospinal fluid exchange in the optic nerve in normal-tension glaucoma. $\mathrm{Br} \mathrm{J}$ Ophthalmol. 2012;96:544-8.

10. Boye D, Montali M, Pircher A, et al. Clin Exp Ophthalmol. 2018; 46:511-8.

11. Pircher A, Montali M, Wostyn P, et al. Impaired cerebrospinal fluid dynamics along the entire optic nerve in normal-tension glaucoma. Acta Ophthalmol. 2018;96:e562-9.

12. Davison H. Physiology of the cerebrospinal fluid. London: J \& A Churchil Ltd; 1967.

13. Hao J, Pircher A, Miller NR, Hsieh J, Remonda L, Killer HE. Cerebrospinal fluid and optic nerve sheath compartment syndrome: a common pathophysiological mechanism in five different cases? Clin Exp Ophthalmol. https://doi.org/10.1111/ceo.13663.

14. Hardy J, Selkoe DJ. The amyloid hypothesis of Alzheimer's disease: progress and problems on the road to therapeutics. Science. 2002;297:353-6.

15. Tarasoff-Conway JM, Carare RO, Osorio RS, et al. Clearance systems in the brain-implications for Alzheimer disease. Nat Rev Neurol. 2015;11:457-70.

16. Lai SW, Lin CL, Liao KF. Glaucoma may be a non-memory manifestation of Alzheimer's disease in older people. Int Psychogeriatr. 2017;1-7.

17. Mancino R, Martucci A, Cesareo $M$, et al. Glaucoma and Alzheimer disease: a single age-related neurodegenerative disease of the brain. Curr Neuropharmacol. 2017;15:1-7.

18. Bauer G, Killer HE, Forrer A, Huber AR, Jaggi GP. Lipocalinlike prostaglandin D synthase (L-PGDS) concentration in aqueous humor in patients with open-angle glaucoma. J Glaucoma. 2014; 23:164-8.

19. Kaeslin MA, Killer HE, Fuhrer CA, Zeleny N, Huber AR, Neutzner A. Changes to the aqueous humor proteome during glaucoma. PloS ONE. 2016;11:e0165314.

20. Killer HE, Hou R, Wostyn P, Meyer P, Pircher A. Pressure and velocity in intraocular and subarachnoid space fluid chambers: an inseparable couple. Eye. 2019;33:343-6.

21. Goel R, Murthy KR, Srikanth SM, et al. Characterizing the normal proteome of human ciliary body. Clin Proteom. 2013;10:9.

22. Rubenstein D, Yin W, Frame M. Biofluid mechanics. 2nd ed. San Diego, USA: Elsevier; 2015.

23. Knepper PA, Samples JR. Glaucoma research and clinical advances. Amsterdam, The Netherlands: Kugler Publications; 2016-2018.

24. Taniike M, Mohri I, Eguchi N, Beuckmann CT, Suzuki K, Urade Y. Perineuronal oligodendrocytes protect against neuronal apoptosis through the production of lipocalin-type prostaglandin D synthase in a genetic demyelinating model. J Neurosci. 2002; 22:4885-96.

25. Liang $\mathrm{X}, \mathrm{Wu} \mathrm{L}$, Hand $\mathrm{T}$, et al. ProstaglandinD2mediatesneuronal protection via the DP1 receptor. J Neurochem. 2005;92:477-86. 